\title{
Bone regenerating effect of surface-functionalized titanium implants with sustained-release characteristics of strontium in ovariectomized rats
}

This article was published in the following Dove Press journal:

International Journal of Nanomedicine

30 May 2016

Number of times this article has been viewed

\author{
Vincent Offermanns' \\ Ole Zoffmann Andersen ${ }^{2}$ \\ Gregor Riede' \\ Inge Hald Andersen ${ }^{3}$ \\ Klaus Pagh Almtoft ${ }^{3}$ \\ Søren Sørensen ${ }^{3}$ \\ Michael Sillassen ${ }^{2}$ \\ Christian Sloth Jeppesen ${ }^{3}$ \\ Michael Rasse' \\ Morten Foss ${ }^{2}$ \\ Frank Kloss' \\ 'Department of Cranio-, Maxillofacial \\ and Oral Surgery, Medical University \\ Innsbruck, Innsbruck, Austria; \\ Interdisciplinary Nanoscience \\ Center (iNANO), Faculty of Science \\ and Technology, Aarhus University, \\ Aarhus, Denmark; ${ }^{3}$ Tribology Centre, \\ Danish Technological Institute, Aarhus, \\ Denmark
}

\begin{abstract}
Since strontium ( $\mathrm{Sr}$ ) is known for its anabolic and anticatabolic effect on bone, research has been focused on its potential impact on osseointegration. The objective of this study was to investigate the performance of nanotopographic implants with a Sr-functionalized titanium (Ti) coating ( $\mathrm{Ti}-\mathrm{Sr}-\mathrm{O}$ ) with respect to osseointegration in osteoporotic bone. The trial was designed to examine the effect of sustained-release characteristics of $\mathrm{Sr}$ in poor-quality bone. Three Ti-Sr-O groups, which differed from each other in coating thickness, Sr contents, and $\mathrm{Sr}$ release, were examined. These were prepared by a magnetron sputtering process and compared to uncoated grade $4 \mathrm{Ti}$. Composition, morphology, and mechanical stability of the coatings were analyzed, and $\mathrm{Sr}$ release data were gained from in vitro washout experiments. In vivo investigation was carried out in an osteoporotic rat model and analyzed histologically, 6 weeks and 12 weeks after implantation. Median values of bone-to-implant contact and new bone formation after 6 weeks were found to be $84.7 \%$ and $54.9 \%$ (best performing Sr group) as compared to $65.2 \%$ and $23.8 \%$ (grade 4 Ti reference), respectively. The 12 -week observation period revealed $84.3 \%$ and $56.5 \%$ (best performing Sr group) and $81.3 \%$ and $39.4 \%$ (grade $4 \mathrm{Ti}$ reference), respectively, for the same measurements. The increase in new bone formation was found to correlate with the amount of Sr released in vitro. The results indicate that sputtered nanostructured $\mathrm{Ti}-\mathrm{Sr}-\mathrm{O}$ coatings showed sustained release of $\mathrm{Sr}$ and accelerate osseointegration even in poor-quality bone, and thus, may have impact on practical applications for medical implants.
\end{abstract}

Keywords: nanotopography, osteoinduction, osseointegration, osteoporosis, rodent

\section{Introduction}

Osteoporosis is characterized by deterioration of bone's microarchitecture caused by an impaired balance between the bone-resorbing osteoclasts and bone-forming osteoblasts. ${ }^{1,2}$ This condition results in a higher susceptibility, eg, femoral neck and vertebral fractures, which are the main clinical consequences. Animals that underwent an ovariectomy (OVX) are a common model for research on osteoporosis, ${ }^{3-5}$ which is also believed to be the concept for other models with impaired bone metabolism with higher risk of implant failure such as radiotherapy ${ }^{6-8}$ or treatment with bisphosphonates, ${ }^{9}$ although these changes underlie other pathomechanisms.

The concept of osseointegration is among the key factors for a successful long-term implant survival, and the resulting interface between bone and metal is responsible for the present-day high success rate of medical implants. ${ }^{10,11}$ In decrepit bone, implant failure is, however, expected to increase, similar to the currently used ovariectomized animal model, where the procedures involved in preparing the model induce
Correspondence: Vincent Offermanns Department of Cranio-, Maxillofacial and Oral Surgery, Medical University Innsbruck, Anichstrasse 35, 6020 Innsbruck, Austria

Tel +435I250424373

Email vincent.offermanns@i-med.ac.at
International Journal of Nanomedicine 2016: | | 243|-2442

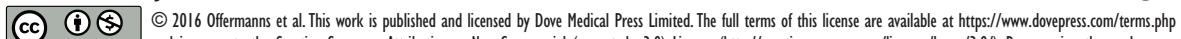

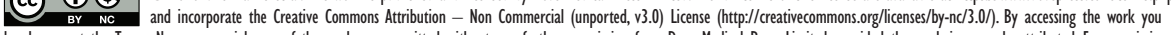
hereby accept the Terms. Non-commercial uses of the work are permitted without any further permission from Dove Medical Press Limited, provided the work is properly attributed. For permision for commercial use of this work, please see paragraphs 4.2 and 5 of our Terms (https:/ / www.doveperess.com/terms.php).

2431
Dovepress

http://dx.doi.org/10.2147/IjN.S101673 
conditions of poor bone quality. The potential of bioactive surfaces with respect to effectiveness on bone metabolism and accelerated anchorage has gained increased research attention over the past couple of years, and the overall results have shown the benefits associated with guided interactions between the implant surface and the environment. Physical, ${ }^{12}$ chemical, ${ }^{13,14}$ and biological ${ }^{15,16}$ modifications as well as topographical changes ${ }^{17-19}$ have been described as potential routes to lead the cellular response toward new bone formation always attempting to reach a successful, long-living bone-to-implant anchorage. ${ }^{20}$ Functionalization of implant surfaces with $\mathrm{Sr}$ is based on the knowledge of its antiosteoporotic properties since it has been used for more than 60 years for the treatment of postmenopausal-related bone loss. Oral administration of $\mathrm{Sr}$, as strontium ranelate (SrRan), has been shown to effectively reduce the risk of newly occurring fractures in postmenopausal women ${ }^{21,22}$ as it affects both the bone-forming osteoblasts and bone-resorbing osteoclasts by interfering in cellular processes and apoptotic events. ${ }^{23-25}$ The mechanisms through which $\mathrm{Sr}$ affects bone remodeling are not yet fully understood. However, a Sr-driven differentiation of human mesenchymal stem cells by activation of canonical and noncanonical Wnt signaling, ${ }^{25,26}$ an inhibitory effect on osteoclast activity, ${ }^{27}$ and an influence on differentiation of osteoclasts and osteoblasts have been observed. ${ }^{23}$ In vivo studies have shown that Sr can enhance osseointegration both in relation to surface modifications and via oral administration of SrRan. ${ }^{28-33}$

With respect to possible clinical applications, relating to modification of medical devices, Sr-enriched applications such as biogenic minerals, bioactive glasses, and bone cement have been reported to have a beneficial effect on mesenchymal stem cell differentiation concerning osteogenesis. ${ }^{34-36}$ This effect has also been observed for Sr-coated titanium (Ti) surfaces. ${ }^{33,37}$

The aim of the current study was to evaluate the performance of Sr-releasing functionalized Ti nanosurfaces in an osteoporotic animal model. The surface modification, which has already been investigated in previous experiments, ${ }^{31,38}$ is based on a magnetron sputtering process that enables fabrication of Ti-based coatings comprising $\mathrm{Sr}$ and oxygen (O) (Ti-Sr-O).

The magnetron sputtering platform allows for preparing coatings with different Sr release profiles ${ }^{31,38}$ with a nanoscaled surface topography. Investigations of the functionalized surfaces were carried out using X-ray photoelectron spectroscopy (XPS), scanning electron microscopy (SEM), white light interferometry, and inductively coupled plasma atomic emission spectroscopy (ICP-AES). The performance of the coatings with respect to new bone formation and osseointegration has been examined in vivo in a rat model, 14 weeks after bilateral OVX, and evaluated 6 weeks and 12 weeks after implantation.

This study shows the performance of Sr-functionalized surfaces, with tailored release properties, under in vivo laboratory conditions, and gives first impressions of the efficacy of the developed coating platform for situations involving compromised bone quality. With its beneficial effect on bone apposition, this modified nanosurface comprising Sr may hold clinical potential within areas of orthopedic and dental implantology.

\section{Materials and methods}

\section{Sample preparation}

Implants with a length of $5 \mathrm{~mm}$ and a diameter of $1.6 \mathrm{~mm}$ were prepared from grade $4 \mathrm{Ti}$ (Elos Medtech Pinol A/S, Gørløse, Denmark). These were manufactured with a selftapping threaded area at the outer $1 \mathrm{~mm}$ implant end and an internal hex for insertion. Unmodified implants served as the control group, while the three experimental groups investigated throughout this study were based on coatings originally having a Sr surface content of 9.1 at $\%$ Sr (Table 1). A magnetron sputtering process was used to synthesize these coatings as described earlier. ${ }^{31,38}$ The deposition process with two different target types (grade $1 \mathrm{Ti}$ and a sintered composite target composed of $50 \% \mathrm{Ti}$ and $50 \% \mathrm{TiSrO}_{3}(\mathrm{w} / \mathrm{w})$ purity $99.99 \%$ ) was conducted with a total target power of $1,000 \mathrm{~W}$ and run at a chamber pressure of $1,100 \mathrm{mPa}$. Subsequently, two of the experimental coatings were modified by washing procedures to reduce the initial burst release of $\mathrm{Sr}$; the

Table I The three Ti-Sr-O coating groups used throughout this study

\begin{tabular}{lllll}
\hline Group \# & Coating thickness & Prewash parameters & Final Sr surface content & Samples description (prewash parameters) \\
\hline $\mathrm{I}$ & $\mathrm{I}, 500 \mathrm{~nm}$ & 22 minutes PBS & $5.8 \pm 0.2 \mathrm{at} \%$ & $\mathrm{I}, 500 \mathrm{~nm}(22 \mathrm{minutes})$ \\
2 & $2,000 \mathrm{~nm}$ & No wash & $9.1 \pm 0.03 \mathrm{at} \%$ & $2,000 \mathrm{~nm}$ (no wash) \\
3 & $2,000 \mathrm{~nm}$ & Industrial wash & $5.2 \pm 0.1 \mathrm{at} \%$ & $2,000 \mathrm{~nm}$ (industrial wash) \\
\hline
\end{tabular}

Notes: The sample name indicates the thickness of the coating and the postdeposition treatment used to modify the sample. Both the I,500 nm and 2,000 nm coatings were deposited using the same conditions but with a reduced process time in the case of the I,500 nm coating. The Sr surface content was determined by XPS.

Abbreviations: at\%, atomic percentage; PBS, phosphate buffered saline; XPS, X-ray photoelectron spectroscopy. 
$1,500 \mathrm{~nm}$ group was placed in phosphate buffered saline (PBS), $\mathrm{pH} 7.4$, at $37^{\circ} \mathrm{C}$ for 22 minutes and, subsequently, briefly rinsed three times in MilliQ water. The two 2,000 nm groups were either 1) left with no further modifications or 2) given an industrial wash in the form of a validated alkaline cleaning, which was routinely used for cleaning implantable devices (Elos Medtech Pinol A/S). The three Ti-Sr-O coating groups are described in Table 1.

\section{Sample characterization}

The thickness of the coatings was evaluated via coated silicon ( $\mathrm{Si}$ ) wafer substrates, which were subsequently cleaved to allow for a cross-sectional view. Investigations were performed with SEM (Nova 600; FEI Company, Eindhoven, the Netherlands), XPS (Kratos Axis UltraDLD; Kratos Analytical Ltd., Manchester, UK), CasaXPS software that analyzed the $\mathrm{O} 1 \mathrm{~s}$, Ti2p, and $\mathrm{Sr} 3 \mathrm{~d}$ peaks, and BW-S501 white light interferometric microscope system (Nikon Corporation, Tokyo, Japan) fitted with a $10 \times$ magnification lens for surface roughness $R_{\mathrm{a}}$ of the implant groups at the submicrometer scale. For the removal of organic contamination, all samples were treated with UV ozone (BioForce Nanosciences, Inc., Ames, IA, USA) for a 30-minute period prior to analysis.

For these investigations, a total of three samples from each group were examined and for each implant four individual measurements were performed, spanning the total length of the subcortical part of the implant (below the thread).

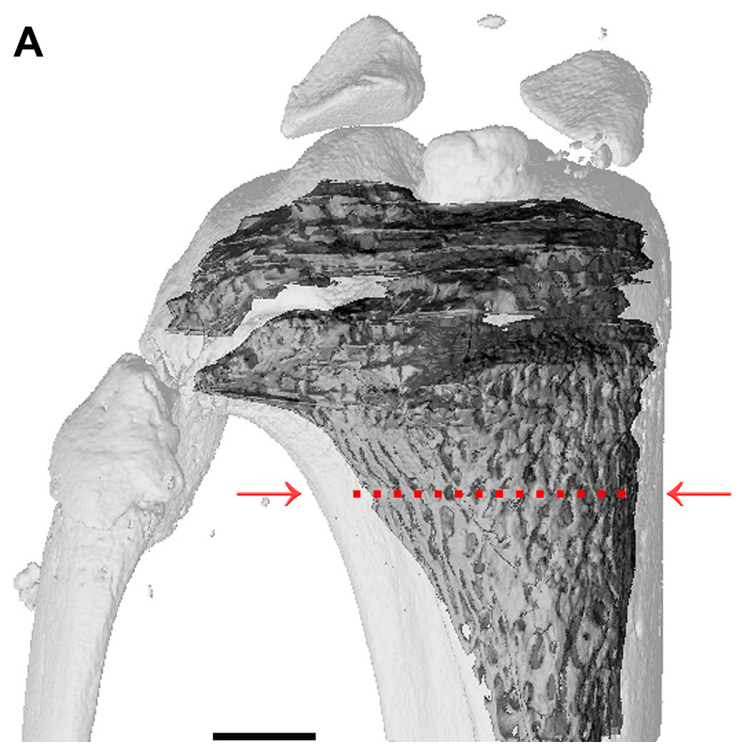

\section{Sr release}

Coatings deposited on implants were used to investigate the Sr release in vitro by dipping the samples in PBS, $\mathrm{pH} 7.4$. The surface area of the samples used was $0.31 \mathrm{~cm}^{2}$, and a total of three samples were used for each sample group. The washout was performed by submerging the samples from each group in $1 \mathrm{~mL}$ PBS in a $2 \mathrm{~mL}$ Eppendorf tube. Samples were then placed at $37^{\circ} \mathrm{C}$ under static conditions. The buffer solutions were collected at intervals of 1, 3, 5, 7 and 14 days, and at each point, the total amount of PBS was collected and replaced by fresh $\mathrm{PBS}$, before the samples were further incubated. ICP-AES (ICP-AES Optima 3300 DV; PerkinElmer Inc., Waltham, MA, USA) was used for the analysis of the Sr content in the collected PBS.

\section{Surgical procedure}

After obtaining permission from the Austrian government (BMWF-66.011/0165-II/3b/2012), 30 female Wistar rats (Charles River $\mathrm{GmbH}^{\circledR}$, Kißlegg, Germany) underwent surgery 14 weeks after bilateral OVX. Micro-computed tomography (CT) images of tibiae from healthy and ovariectomized animals are shown in Figure 1. The animals were used in compliance with the institutional animal health care regulations and animal experimental procedures were approved by the Institutional Animal Care and Use Committee (Central Laboratory Animal Facilities, Medical University Innsbruck). At the time of surgery, the rats weighed $\sim 300 \mathrm{~g}$. Prior to initiating the procedure, all implants were sterilized by gamma irradiation. After a

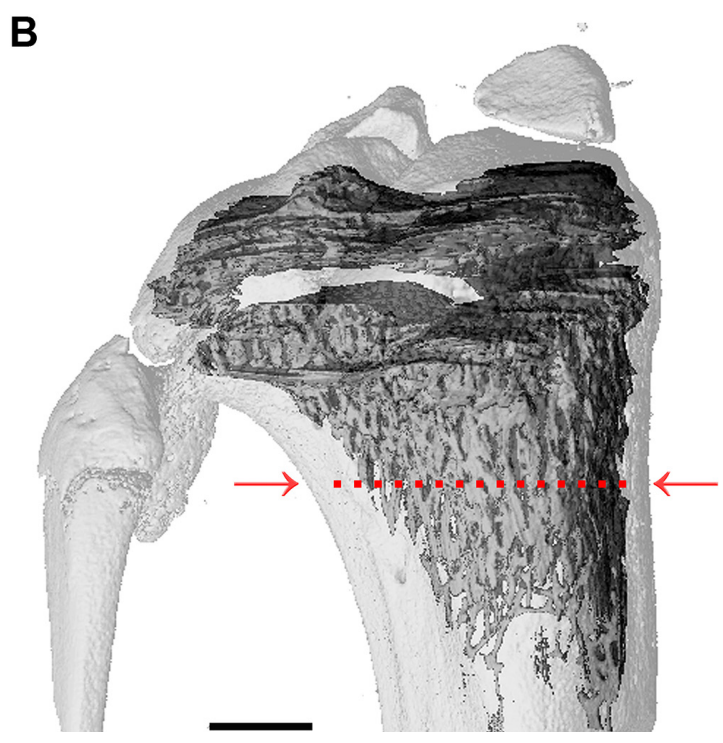

Figure I Micro-computed tomography images.

Notes: (A) Tibia control and (B) tibia from ovariectomized rat (OVX) 14 weeks after bilateral OVX. The tibia from the OVX rat shows $\sim 50 \%$ less bone volume compared to control; measurements were taken $2 \mathrm{~mm}$ away from the epiphyseal plate (indicated by red arrows and dotted lines). Scale bars are I,000 $\mu \mathrm{m}$. Abbreviation: OVX, ovariectomy. 
14-day acclimatization period, all rats received two implants, one in each proximal tibia, as described in the literature. ${ }^{39}$

Anesthesia was initialized with Isoflurane (Forane ${ }^{\circledR}$; Abbott Laboratories, Abbott Park, IL, USA) with subsequent intramuscular administration of Ketaminol (Ketamin ${ }^{\circledR}$; Dr E Graeub AG, Bern, Switzerland; $100 \mathrm{mg} / \mathrm{kg}$ body weight) and Xylasol (Xylazin ${ }^{\circledR}$; Dr E Graeub AG; $10 \mathrm{mg} / \mathrm{kg}$ body weight) to assure sufficient narcosis. An overview of the surgical procedure is shown in Figure 2.
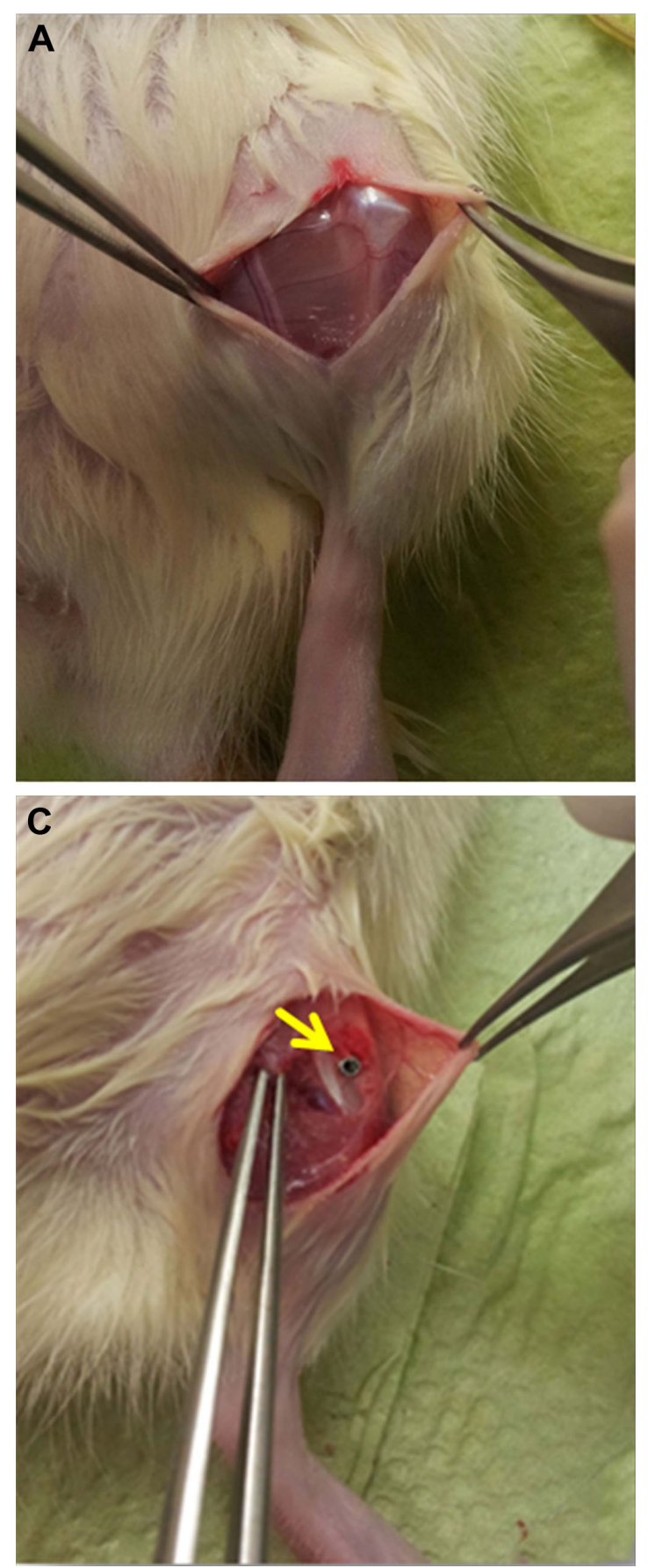

Shaving and sterile washing were employed to perform surgery under aseptic conditions. Access to the tibia was gained by a $10-15 \mathrm{~mm}$ incision followed by repelling of the connective tissue. Drilling was conducted with a bur of $1.6 \mathrm{~mm}$ in diameter (Electric Pen Drive System; DePuy Synthes, Zuchwil, Switzerland), while the operation area was cooled with isotonic saline solution (Sigma-Aldrich Co., St Louis, MO, USA). Implants were directly inserted due to the self-tapping threaded area at the implant end.
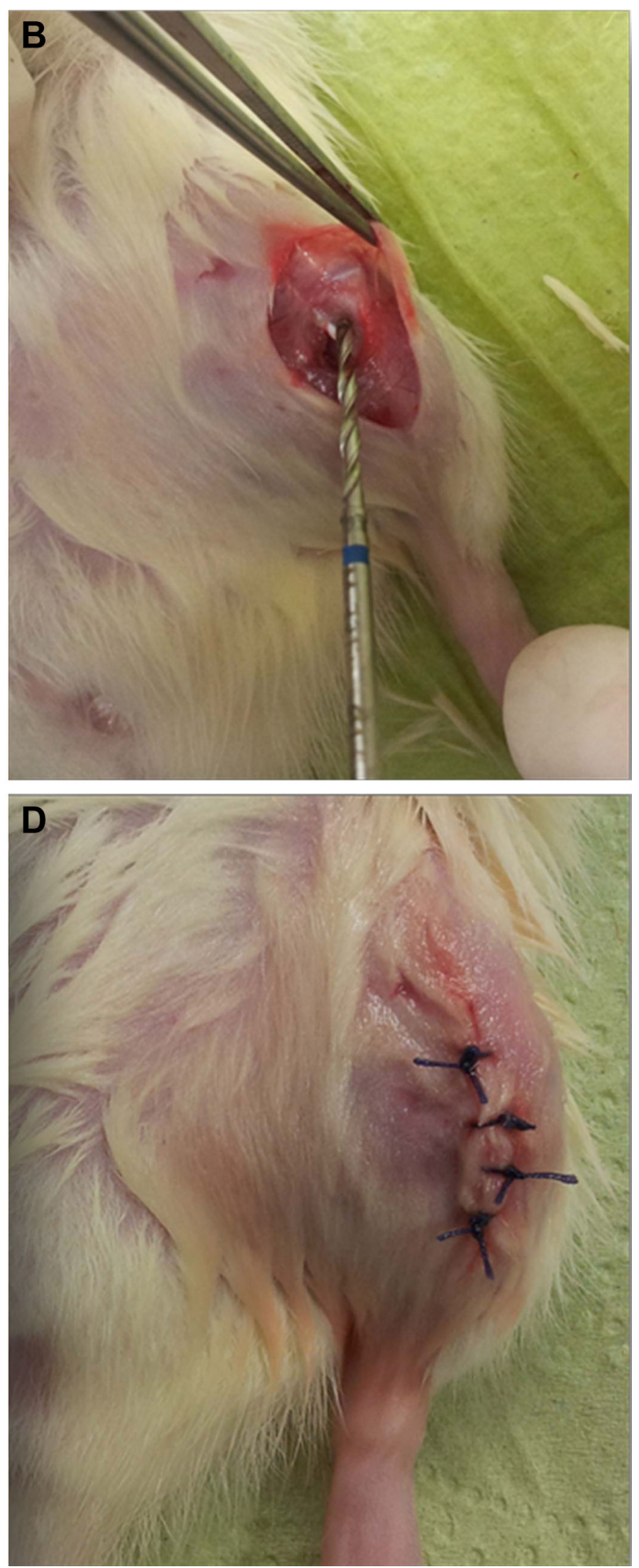

Figure 2 Surgical procedure shown in four steps.

Notes: (A) Exposed knee cap after incision; (B) drill between the medial collateral ligament and patella tendon; (C) inserted implant (marked with yellow arrow); and (D) skin suture. 
After implantation, suturing was performed with Vicryl 4-0 (Ethicon Products, Norderstedt, Germany). Subsequently, analgesic subcutaneous injection of Carprofen (Rimadyl ${ }^{\circledR}$; Pfizer, Inc., New York, NY, USA; 4 mg/kg body weight) was administered, and oral antibiotics (Enrofloxacin; (Baytril ${ }^{\circledR}$; Bayer AG, Leverkusen, Germany; $100 \mathrm{mg} / \mathrm{mL}$ ) were given in the drinking water for a period of 3 days.

Animals were kept in individual cages supplied with standard laboratory food and tap water ad libitum for either 6 weeks or 12 weeks. After these time periods, the animals were euthanized utilizing $\mathrm{CO}_{2}$ gas with subsequent cervical fracturing to ensure termination of vital functions. Subsequent to harvesting the osseous samples, the abdominal cavity was opened to verify that the OVX had occurred.

\section{Histological evaluation}

Histological slides were processed following the method of Donath and Breuner. ${ }^{40}$ Briefly, samples were cut using a precision saw with a blade width of $0.1 \mathrm{~mm}$ (Exakt 300; Exakt, Oklahoma City, OK, USA). After the slides were split along the implant axis, samples were bound to a microscope slide to allow for secondary cutting, yielding a thickness of $\sim 100 \mu \mathrm{m}$. Subsequently, grinding (grit sizes $1,000,2,500$, and 4,000) was performed to achieve a final sample thickness of $60 \mu \mathrm{m}$. The histological preparation was completed by polishing all samples using Micropolish II, $1.0 \mu \mathrm{m}$ (Buehler, Esslingen am Neckar, Germany) before staining with toluidine blue.

Evaluation of new bone formation and bone-to-implant contact (BIC; magnification $40 \times$ ), according to previous investigations, was performed with NIS Elements BR 3.10 software (Nikon Corporation). ${ }^{31,38}$ A standard rectangular area was defined by measuring 3,000 $\mu \mathrm{m}$ along the axis of the implant and extending $250 \mu \mathrm{m}$ orthogonal to the surface of the implant (Figure 3). This box was used as a reference area for the analysis of de novo bone synthesis by using the area tool of the software. Evaluation areas were at least at a $2 \mathrm{~mm}$ distance from the epiphyseal plate.

Results were then expressed as a percentage of new bone formation within the standard rectangular area and a percentage of direct contact between new bone and implant along the $3,000 \mu \mathrm{m}$ reference.

\section{Statistical analysis}

Data are presented as median value \pm interquartile range. The analysis for statistical significance in relation to the in vivo experiments was performed using the Mann-Whitney $U$-test with confidence intervals of $95 \%, 99 \%$, and $99.9 \%$ indicated by $* P<0.05, * * P<0.01, * * * P<0.001$, and ${ }^{\dagger}(P<0.05)$, respectively (the three confidence intervals are indicated by four different symbols as $P<0.05$ is shown with both * and ${ }^{\dagger}$ symbols to clarify that different groups are being referred to). SPSS for Windows ${ }^{\circledR}$ 15.0.1 (SPSS Inc., Chicago, IL, USA) and Microsoft Excel 2010 (Microsoft Corporation, Redmond, WA, USA) were used for statistical analysis.

\section{Results}

The surface content of Sr, analyzed with XPS, was found to vary between the experimental groups. The elemental composition of the samples is summarized in Table 2.

Coating thickness and morphology were evaluated by SEM. Figure 4 presents cross-sectional views of the coatings. The effective $\mathrm{Ti}-\mathrm{Sr}-\mathrm{O}$ coating thickness is $\sim 1,500 \mathrm{~nm}$ and $2,000 \mathrm{~nm}$. The morphology of the two coatings is characterized by column-like structures having a granular and porous appearance. This structure has been found to yield a high effective surface area that is important for obtaining the desired release characteristics, eg, elevated long-term release of Sr. Neither thickness nor morphology changes can be seen with SEM after washing the samples as described in earlier investigations. ${ }^{41}$

Data from the $\mathrm{Sr}$ release performed on the three different implant groups are presented in Figure 5. The washing procedures (22 minutes and industrial wash) are found to reduce the initial burst release significantly.

Additionally, we observed that this procedure not only affects the washout within the first 24 hours but also causes differences in the amount of $\mathrm{Sr}$ released over the full 14-day range.

The surface roughness of the implants used for the study was characterized at the submicrometer level in order to analyze whether the in vivo results were likely to have been influenced by morphological variations caused by the coating process. The results from this roughness analysis are presented in Figure 6.

The in vivo experiment was carried out to investigate the effect of the different coatings on bone formation in compromised bone. For the specific study, an ovariectomized rat model, mimicking osteoporotic conditions, was utilized. During the implantation period, three animals were sacrificed after 1 day and 2 days, respectively, due to untreatable wounds. For all other animals, healing was uneventful. No allergic reactions or infections were observed.

Figure 3 shows the images of exemplary histological slides for the 2,000 $\mathrm{nm}$ group with the highest Sr release and the grade 4 Ti reference after 6 weeks and 12 weeks, respectively. This figure also includes an illustration of the reference area used for evaluating the new bone formation and BIC.

Quantitative results for new bone formation and osseointegration were expressed as bone area percentage (BA\%) 

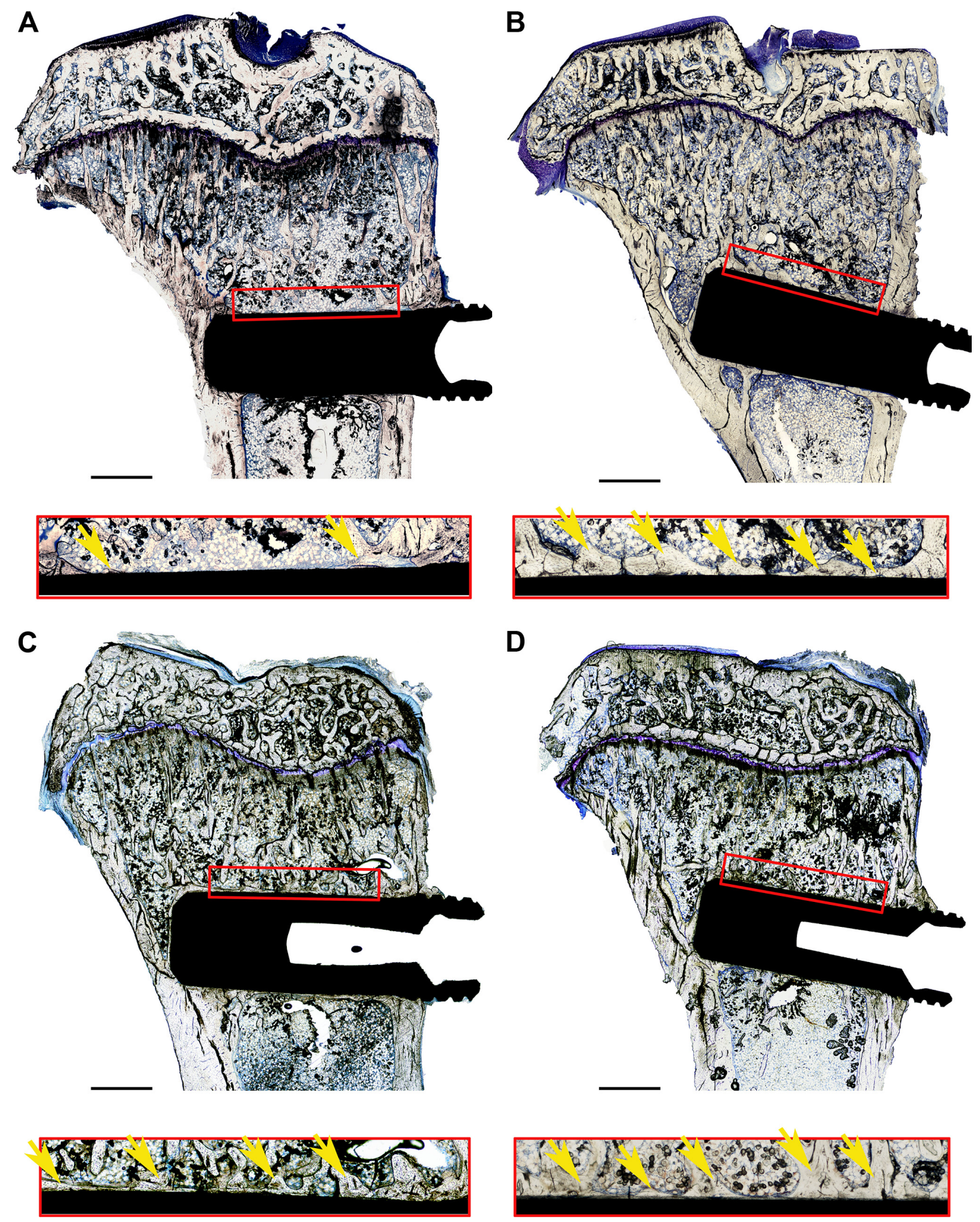

Figure 3 Histological samples, stained with toluidine blue, including reference boxes and magnified images.

Notes: Reference box was $250 \times 3,000 \mathrm{~mm}^{2}$ and marks the standard area used to evaluate new bone formation: (A) 6 weeks, grade 4 Ti reference; (B) 6 weeks, unmodified 2,000 nm Ti-Sr-O coating; (C) 12 weeks, grade 4 Ti reference; and (D) 12 weeks, unmodified 2,000 nm Ti-Sr-O coating. The area of new bone formation inside the reference box was measured for all samples and used to calculate the percentage of de novo bone synthesis (marked with yellow arrows) with respect to the total reference area. In all cases, the edge of the reference area was at least $2 \mathrm{~mm}$ away from the growth plate. The side of the reference box facing the implant surface was also used to evaluate the percentage of direct BIC with respect to the total length of the reference. Scale bars are I,000 $\mu \mathrm{m}$.

Abbreviation: BIC, bone-to-implant contact.

and $\mathrm{BIC}$ percentage $(\mathrm{BIC} \%)$. The results for the 6-week and 12-week groups are presented in Figures 7 and 8, respectively.

Significant differences could be observed for the comparison of the two time intervals for the grade 4 Ti reference $(P=0.01)$ and industrial wash $(P=0.03)$, whereas groups with higher release kinetics revealed almost same amounts of bone apposition and BIC (Figure 9).

\section{Discussion}

The purpose of this study was to investigate the effect of sustained release of $\mathrm{Sr}$ from nanostructured Ti surfaces in relation 
Table 2 Elemental composition of the surfaces of the tested coatings expressed as at\%

\begin{tabular}{lllll}
\hline Group \# & $\begin{array}{l}\text { Samples description } \\
\text { (washing parameters) }\end{array}$ & Ti (at\%) & Sr (at\%) & O (at\%) \\
\hline I & I,500 $\mathrm{nm}$ (22 minutes) & $24.3 \pm 0.3$ & $5.8 \pm 0.2$ & $69.9 \pm 0.5$ \\
2 & $2,000 \mathrm{~nm}$ (no wash) & $22.2 \pm 0.5$ & $9.1 \pm 0.03$ & $68.6 \pm 0.5$ \\
3 & $2,000 \mathrm{~nm}$ (industrial wash) & $24.8 \pm 0.5$ & $5.2 \pm 0.1$ & $70.0 \pm 0.3$ \\
\hline
\end{tabular}

Note: It is evident that the washing procedures reduce the surface $\mathrm{Sr}$ content of the two untreated coatings.

Abbreviation: at\%, atomic percentage.

to osseointegration in osteoporotic-like bone. Although success rates of medical implants, eg, total hip arthroplasty ${ }^{11}$ and dental root replacement, ${ }^{10}$ are considered high, patients with poor bone quality are believed to be predisposed for higher implant failure rates. ${ }^{42-44}$ The ovariectomized rat model is frequently utilized due to easy handling, simple operation procedure, and predictable effects on the bone structure. ${ }^{3-5}$ $\mathrm{Sr}$ is known for its beneficial effects on bone remodeling, which has been investigated in numerous in vitro, in vivo, and clinical studies. ${ }^{22,25,31,37,45-47}$ Meunier et al were able to show a risk reduction of $41 \%$ for new vertebral fractures in postmenopausal women after treatment with SrRan in a prospective, randomized, double-blind, placebo-controlled trial. ${ }^{21}$ With respect to implantology, Li et al observed that beneficial effects of both $\mathrm{Sr}$ and SrRan in vivo as bone formation and push-out forces were found to be increased in the test groups. ${ }^{28,30}$ Interestingly, chemically modified surfaces with $\mathrm{Sr}$ ions also revealed higher torque removal values for Sr-enriched surfaces when compared to a hydrophilic surface (SLActive ${ }^{\mathrm{TM}}$ ), following a 2-week implantation period. ${ }^{37}$

We recently reported on magnetron sputtered $\mathrm{Ti}-\mathrm{Sr}-\mathrm{O}$ implant coatings and their Sr-release kinetics resulting in increased BIC and de novo bone synthesis in healthy bone..$^{31,38}$ Hence, we hypothesized that a Sr-functionalized surface could also affect bone apposition in the tibia of ovariectomized rats, mimicking osteoporotic-like bone behavior.

Within a 6-week period, new bone formation was found to be $54.9 \%$ for the best performing group correlating with the highest amount of $\mathrm{Sr}$ released in vitro $(2,000 \mathrm{~nm} \mathrm{Ti}-\mathrm{Sr}-\mathrm{O}$ group), while the Ti control group revealed a value of $23.8 \%$ $(P<0.001)$. BIC \% was significantly increased in the 9.1 at $\%$ and 5.8 at $\% \mathrm{Sr}$ in comparison to the Ti control.

Following 12 weeks of implantation, statistical significant differences were present between the investigated Sr groups and the Ti reference with respect to $\mathrm{BA} \%$. $\mathrm{BIC} \%$ was increased in the Ti reference group, while no increase was found in the Sr-modified groups except for the samples with the industrial wash, indicating completion of the osseointegration process prior to the 6-week time point.

The observation that significant differences exist between the grade 4 Ti reference and the $\mathrm{Ti}-\mathrm{Sr}-\mathrm{O}$ groups, with respect to $\mathrm{BA} \%$, following 12 weeks of implantation is likely related to the osteoinductivity and stability of the Sr ion. According to Habibovic and de Groot, osteoinductive biomaterials possess a higher ability to instruct their in vivo surroundings to form new bone than nonosteoinductive biomaterials and should hence be favorable. ${ }^{48}$ Although the $\mathrm{Sr}$ ion is prone to be incorporated into bone, it is not degraded over time. This could be advantageous compared to the inherent lability, eg, cells or proteins, when functionalized surfaces are inserted into a biologic environment. This is the case as approaches for delivery of biologically derived osteoinductive components are associated with potential challenges concerning the longterm stability of the active components. ${ }^{49}$ The decomposable nature of proteins infers that a delivery system needs to protect the active substance prior to its release. For instance, it has been found that $<5 \%$ of the total amount of bone morphogenetic protein (BMP) remains at the application site when
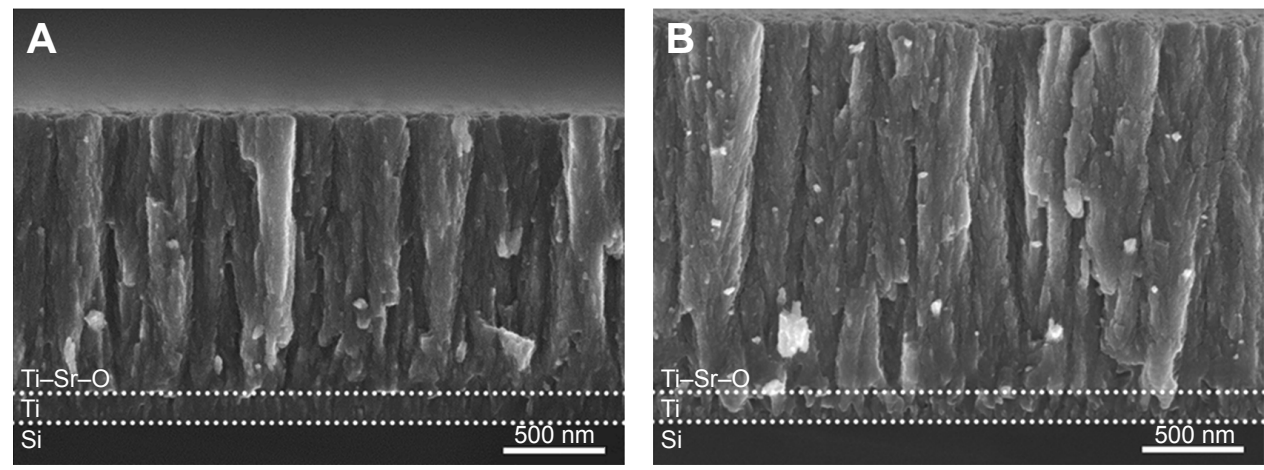

Figure 4 SEM images of the two basis coatings deposited onto Si wafer substrates.

Notes: The intermediate Ti binding layer and Ti-Sr-O layer are indicated by the dotted lines: (A) 1,500 nm Ti-Sr-O coating. (B) 2,000 nm Ti-Sr-O coating. The morphology of the two coatings appears highly similar, having a column-like structure. Moreover, the structure appears granular and porous, and this has been found to be a key feature in relation to obtaining the desired Sr release characteristics.

Abbreviation: SEM, scanning electron microscopy. 
A

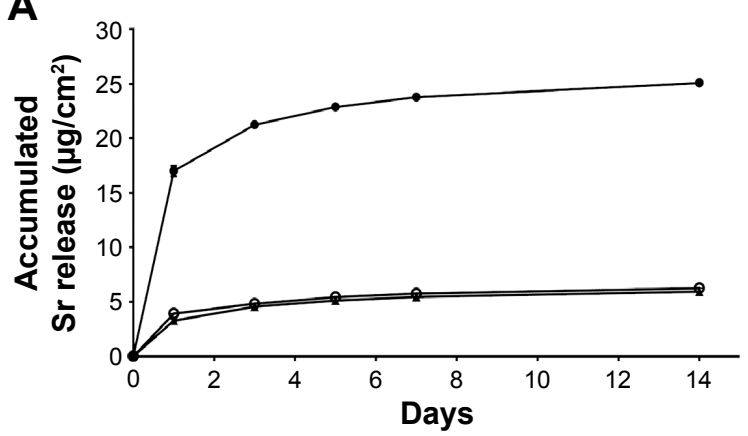

B

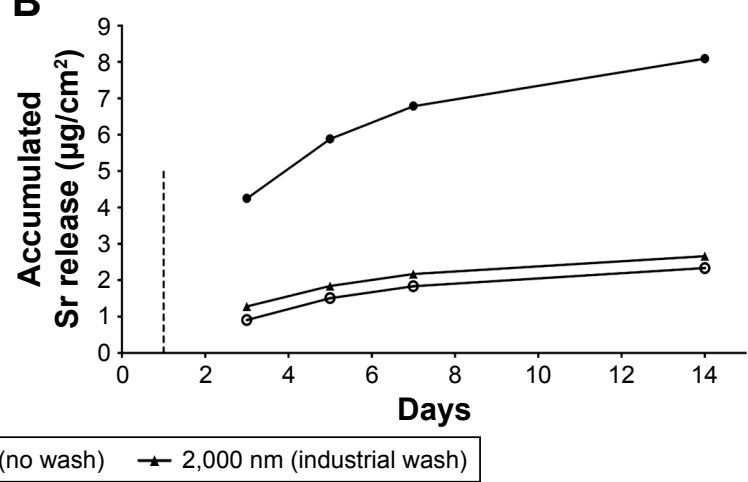

Figure 5 Accumulated $\mathrm{Sr}$ release from the three Ti-Sr-O groups as determined by ICP-AES.

Notes: (A) The accumulated amount of Sr released over a period of I4 days. It is evident that the 2,000 nm (no wash) coating presents the highest release value, while the release of the $2,000 \mathrm{~nm}$ (industrial wash) and the 1,500 $\mathrm{nm}$ (22 minutes) is significantly lower. By presenting the accumulated release, the amount released within the first day greatly influences the graphical representation. (B) The accumulated amount of Sr release in the period between days I and I4. Here, the data from the initial 24 hours are omitted as indicated by the dashed line. Standard deviations for the measured values are included. However, for the majority of the measurements, these are smaller than the utilized markers. The legend applies to both graphs.

Abbreviation: ICP-AES, inductively coupled plasma atomic emission spectroscopy.

supplied directly, whereas combinations of the proteins with gelatin foam or collagen showed increased retention ranging from $15 \%$ to $55 \%$ in a 4 -week and 8 -week observation period. ${ }^{49}$ Hence, delivery systems should be biocompatible with sustainable retention of bioactive elements.

With regard to clinical applications, Kloss et al investigated the effect of BMP-2 immobilization on nano-crystalline diamond-coated Ti screws in irradiated pig mandibles. The authors observed an initial increased bone contact ratio. However, no sustainable effect compared with osseointegration in nonirradiated bone could be detected..$^{50}$

Considering the time intervals in the current study, sacrification 12 weeks after implant insertion in ovariectomized rats is described in the literature. ${ }^{28,30} \mathrm{~A}$ 6-week time interval in the described experiments was chosen in order to allow for obtaining data with respect to relatively early osseointegration. It is evident that $\mathrm{BIC}$ for the best performing $\mathrm{Ti}-\mathrm{Sr}-\mathrm{O}$ group after 6 weeks is comparable to the results from the

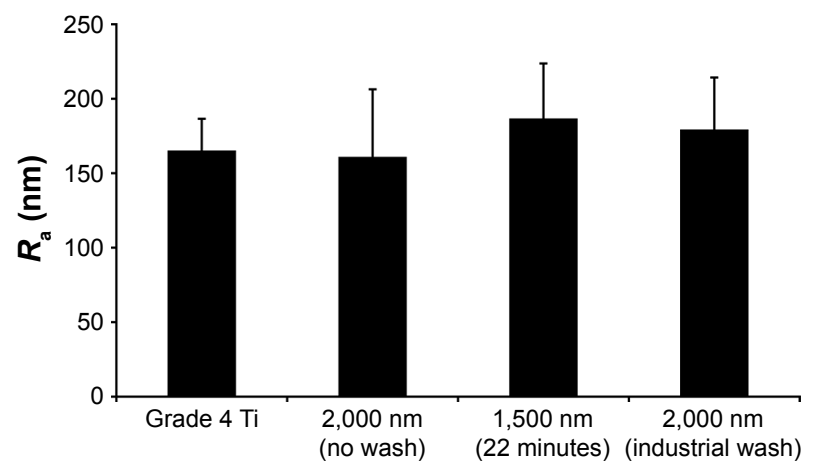

Figure $6 R_{\mathrm{a}}$ measured on the surface of the four different implant groups. Note: No statistical significant differences between the groups were found. Abbreviation: $R_{\mathrm{a}}$, surface roughness. same group after 12 weeks. This indicates that the $\mathrm{Ti}-\mathrm{Sr}-\mathrm{O}$ implants achieve maximum osseointegration at an earlier stage and, eg, it would have been interesting to collect data on early bone response even earlier such as from a 4-week observation period.

The increase in new bone formation of the Srfunctionalized implants was found to correlate with the amount of Sr released into PBS with significant differences regarding new bone formation in comparison to a grade $4 \mathrm{Ti}$ reference, which was also observed in earlier experiments. ${ }^{31,38}$ The release data showed that the industrial cleaning results in release values comparable to those found for the $1,500 \mathrm{~nm}$ (22) coating, having a slightly higher release at the 24-hour time point. Nonetheless, the comparable slopes of the curves underline the similarities between the two coatings. Deterioration of the bone microstructure due to, eg, osteoporosis, radiation therapy, or systemic treatment with bisphosphonates is frequently encountered in the clinic and, with respect to maxillofacial and orthopedic surgery, might affect the survival rate of Ti implants. ${ }^{6,7,44}$

Noble et al reported on an insufficient fit of cementless femoral prostheses, ${ }^{51}$ while Moritz et al pointed out the possible risk of implant migration in poor bone quality. ${ }^{52}$

Buddula et al describes a greater risk of implant failure, when placed in irradiated bone. ${ }^{7}$ Prospectively, this could be relevant in terms of patients receiving irradiation due to cancer treatment. These patients are running a higher risk of implant failure, especially when the implant is placed in grafted bone. ${ }^{6}$ On that account, irradiated animals could serve as models for experimental setups in order to gain knowledge about the performance of implant surfaces, functionalized 
A

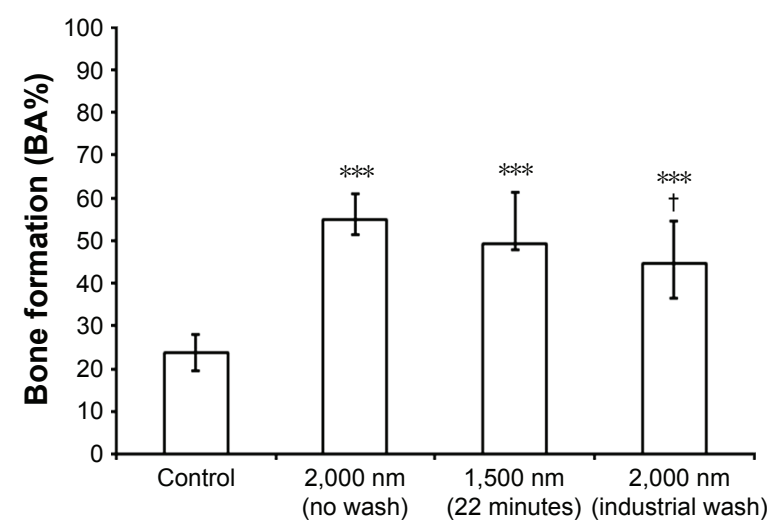

B

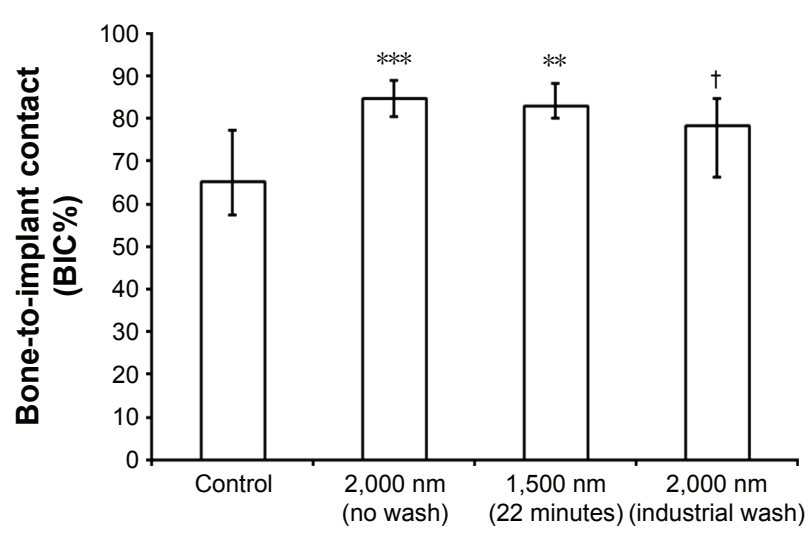

Figure 7 In vivo data 6 weeks after implantation.

Notes: (A) Percentage of new bone formation (BA\%). (B) Percentage of direct bone-to-implant contact (BIC\%). Significant differences with respect to the grade 4 Ti reference are marked with $* * P<0.01$ and $* * * P<0.00$ I. Significant differences with respect to 2,000 nm with no wash are marked with ${ }^{\dagger} P<0.05$.

Abbreviations: BA\%, bone area percentage; $\mathrm{BIC} \%$, bone-to-implant contact percentage.

A

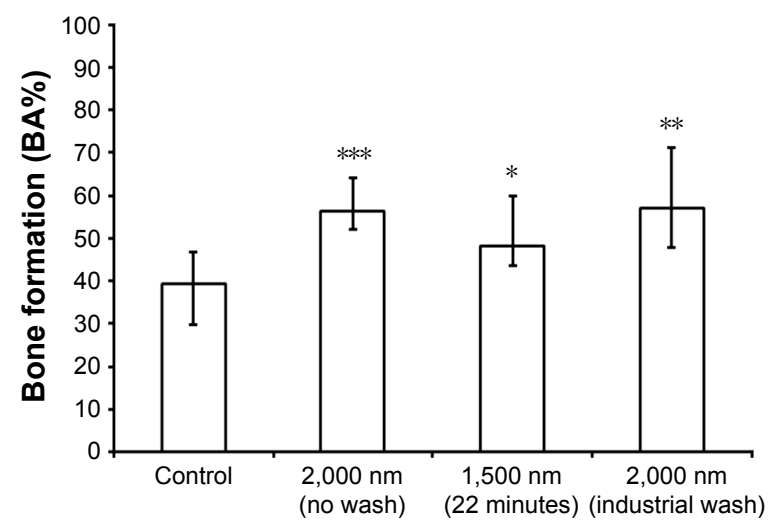

B

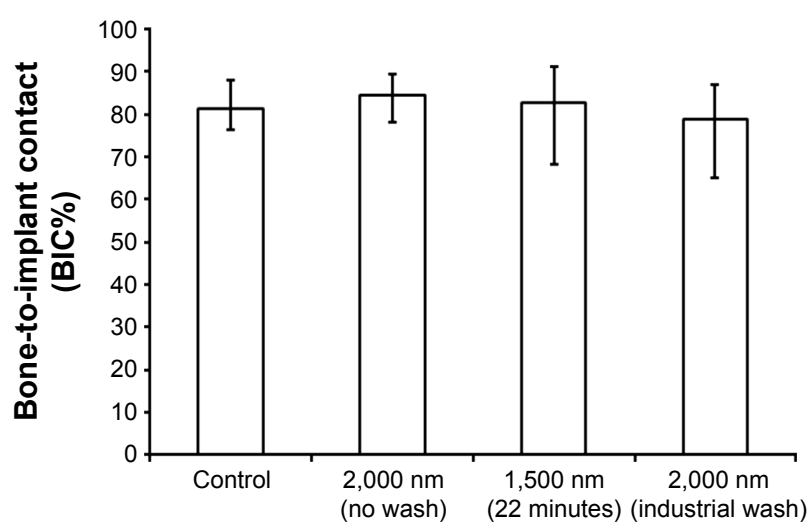

Figure 8 In vivo data 12 weeks after implantation.

Notes: (A) Percentage of new bone formation (BA\%). (B) Percentage of direct BIC. Significant differences with respect to grade $4 \mathrm{Ti}$ reference are marked with $* P<0.05$, $* * P<0.01$, and $* * * P<0.001$.

Abbreviations: $\mathrm{BA} \%$, bone area percentage; $\mathrm{BIC} \%$, bone-to-implant contact percentage.

A

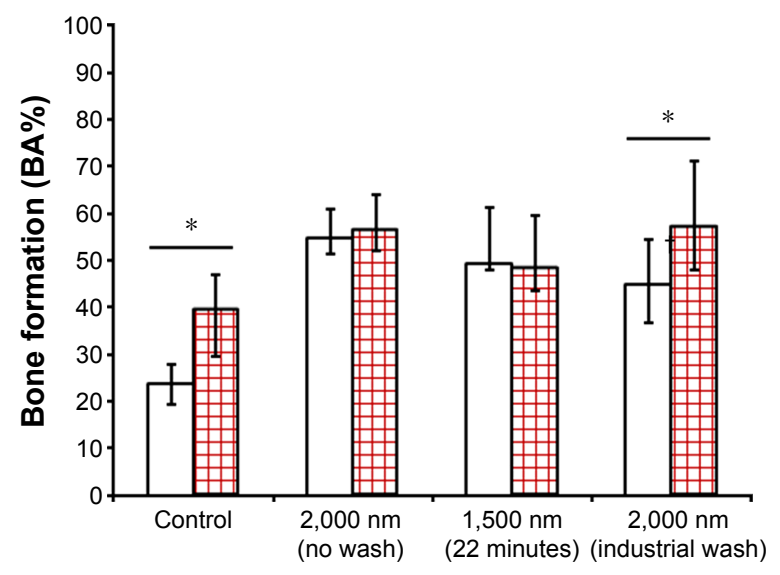

B

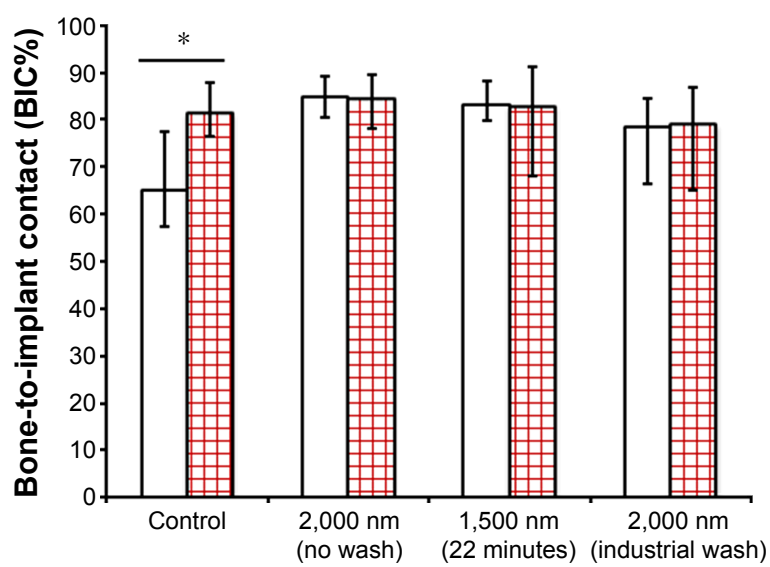

Figure 9 Comparison of (A) BA\% and (B) BIC\% after 6 weeks (white column) and 12 weeks (red squared column) postimplantation.

Note: Significant differences between the two time intervals are marked with $* P<0.05$.

Abbreviations: $\mathrm{BA} \%$, bone area percentage; $\mathrm{BIC} \%$, bone-to-implant contact percentage. 
with active elements, such as $\mathrm{Sr}$, when implanted in compromised bone.

The current study is the first investigation on a magnetronsputtered Sr-functionalized surface within the nanometer topography in an osteoporotic animal model and reveals insights into utilizing Sr for promoting early bone formation in deteriorated bone. Since a large part of the growing population is affected by osteoporosis or related conditions, the results from the current study indicate the potential of the developed technology in relation to, eg, trauma, orthopedic, and maxillofacial surgery. Nonetheless, any predictions on the clinical behavior in human bone should be made restrainedly since the physiological conditions are different than in an experimental setup such as the ovariectomized animal model utilized for the current study.

The physical vapor deposition process as a platform for generating Sr-functionalized coatings is highly tunable with respect to Sr content and in vitro release kinetics. ${ }^{41}$ Furthermore, the data from the current and earlier studies suggest that the Sr release also takes place in vivo. The described coating procedure is considered to be responsible for the sustainable release of Sr with standard deviations $<1 \%$. To our knowledge, measurable values for $\mathrm{Sr}$ released from implant surfaces lack data and a tailored release could be relevant with respect to, eg, cementless hip prostheses stimulating early bone response.

Although standard protocols include early mobilization of patients treated with hip prostheses, dental implants are considered to osseointegrate over time without loading. Hence, Sr-doped surfaces with sustained-release characteristics could be advantageous supporting mechanical anchorage for both applications: enhancing fixation of early loading and acceleration of osseointegration. Moreover, the described surface topography might be favorable with respect to prevention of bacterial adhesion and subsequent peri-implantitis.

The results encourage further research as they highlight the potential benefits of developing functionalized surfaces with sustained-release properties to stimulate, eg, periimplant bone formation and early mechanical anchorage.

The overall results of the current study indicate that the magnetron-sputtere $\mathrm{Ti}-\mathrm{Sr}-\mathrm{O}$ coatings might be interesting for endosseous medical devices, enhancing early osseointegration as well as yielding improved stability also for patients with compromised bone quality.

\section{Conclusion}

The diametral effect of $\mathrm{Sr}$ is known and examined in numerous investigations. The approach to enhance early and late osseointegration in compromised bone presented in this study is based on predictable release characteristics of $\mathrm{Sr}$ from magnetron-sputtered surface coatings. Results indicate a potential benefit of a nanostructured $\mathrm{Ti}-\mathrm{Sr}-\mathrm{O}$ surface with controllable release properties of $\mathrm{Sr}$ in bone of diminished density and, thus, suggest a possible therapeutic potential for traumatologists and orthopedic and maxillofacial surgeons.

The development of a functionalized surface with nanotopography, here with local administration of $\mathrm{Sr}$, might be a possible approach for enhancing osseointegration in compromised bone.

\section{Acknowledgments}

The authors would like to thank Professor Hermann Dietrich from the Central Laboratory Animal Facility at the Medical University Innsbruck for the cooperative work with regard to the in vivo experiments. This research was carried out in collaboration with Elos Medtech Pinol A/S and the Danish Technological Institute and has been supported by the Danish National Advanced Technology Foundation through the project "Strontium functionalized Ti implants" and by the Danish Agency for Science, Technology and Innovation through a mobility scholarship.

\section{Disclosure}

The authors report no conflicts of interest in this work.

\section{References}

1. Mulder JE, Kolatkar NS, LeBoff MS. Drug insight: existing and emerging therapies for osteoporosis. Nat Clin Pract Endocrinol Metab. 2006;2(12):670-680

2. Beppu K, Kido H, Watazu A, Teraoka K, Matsuura M. Peri-implant bone density in senile osteoporosis-changes from implant placement to osseointegration. Clin Implant Dent Relat Res. 2013;15(2):217-226.

3. Jee WS, Yao W. Overview: animal models of osteopenia and osteoporosis. J Musculoskelet Neuronal Interact. 2001;1(3):193-207.

4. Lelovas PP, Xanthos TT, Thoma SE, Lyritis GP, Dontas IA. The laboratory rat as an animal model for osteoporosis research. Comp Med. 2008; 58(5):424-430

5. Wen B, Zhu F, Li Z, Zhang P, Lin X, Dard M. The osseointegration behavior of titanium-zirconium implants in ovariectomized rabbits. Clin Oral Implants Res. 2014;25(7):819-825.

6. Yerit KC, Posch M, Seemann M, et al. Implant survival in mandibles of irradiated oral cancer patients. Clin Oral Implants Res. 2006;17(3): 337-344.

7. Buddula A, Assad DA, Salinas TJ, Garces YI, Volz JE, Weaver AL. Survival of dental implants in irradiated head and neck cancer patients: a retrospective analysis. Clin Implant Dent Relat Res. 2012;14(5): 716-722.

8. Zheng M, Li L, Tang Y, Liang XH. How to improve the survival rate of implants after radiotherapy for head and neck cancer? J Periodontal Implant Sci. 2014;44(1):2-7.

9. Kim I, Ki H, Lee W, Kim H, Park JB. The effect of systemically administered bisphosphonates on bony healing after tooth extraction and osseointegration of dental implants in the rabbit maxilla. Int J Oral Maxillofac Implants. 2013;28(5):1194-1200.

10. Buser D, Janner SF, Wittneben JG, Bragger U, Ramseier CA, Salvi GE. 10-year survival and success rates of 511 titanium implants with a sandblasted and acid-etched surface: a retrospective study in 303 partially edentulous patients. Clin Implant Dent Relat Res. 2012;14(6):839-851. 
11. Mäkelä KT, Matilainen M, Pulkkinen P, et al. Failure rate of cemented and uncemented total hip replacements: register study of combined Nordic database of four nations. BMJ. 2014;348:f7592.

12. Kloss FR, Gassner R, Preiner J, et al. The role of oxygen termination of nanocrystalline diamond on immobilisation of BMP-2 and subsequent bone formation. Biomaterials. 2008;29(16):2433-2442.

13. Schwarz F, Wieland M, Schwartz Z, et al. Potential of chemically modified hydrophilic surface characteristics to support tissue integration of titanium dental implants. J Biomed Mater Res B Appl Biomater. 2009; 88(2):544-557.

14. Lee JK, Choi DS, Jang I, Choi WY. Improved osseointegration of dental titanium implants by $\mathrm{TiO} 2$ nanotube arrays with recombinant human bone morphogenetic protein-2: a pilot in vivo study. Int J Nanomedicine. 2015;10:1145-1154.

15. Yazici H, Fong H, Wilson B, et al. Biological response on a titanium implant-grade surface functionalized with modular peptides. Acta Biomater. 2013;9(2):5341-5352.

16. Zhou Y, Snead ML, Tamerler C. Bio-inspired hard-to-soft interface for implant integration to bone. Nanomedicine. 2015;11(2):431-434.

17. Wennerberg A, Albrektsson T. On implant surfaces: a review of current knowledge and opinions. Int J Oral Maxillofac Implants. 2010; 25(1):63-74.

18. Ballo A, Agheli H, Lausmaa J, Thomsen P, Petronis S. Nanostructured model implants for in vivo studies: influence of well-defined nanotopography on de novo bone formation on titanium implants. Int J Nanomedicine. 2011;6:3415-3428.

19. Jimbo R, Coelho PG, Bryington M, et al. Nano hydroxyapatite-coated implants improve bone nanomechanical properties. J Dent Res. 2012; 91(12):1172-1177.

20. Chappuis V, Buser R, Brägger U, Bornstein MM, Salvi GE, Buser D. Long-term outcomes of dental implants with a titanium plasma-sprayed surface: a 20-year prospective case series study in partially edentulous patients. Clin Implant Dent Relat Res. 2013;15(6):780-790.

21. Meunier PJ, Roux C, Seeman E, et al. The effects of strontium ranelate on the risk of vertebral fracture in women with postmenopausal osteoporosis. N Engl J Med. 2004;350(5):459-468.

22. Reginster JY, Seeman E, De Vernejoul MC, et al. Strontium ranelate reduces the risk of nonvertebral fractures in postmenopausal women with osteoporosis: Treatment of Peripheral Osteoporosis (TROPOS) study. J Clin Endocrinol Metab. 2005;90(5):2816-2822.

23. Marie PJ. Strontium ranelate in osteoporosis and beyond: identifying molecular targets in bone cell biology. Mol Interv. 2010;10(5): 305-312.

24. Braux J, Velard F, Guillaume C, et al. A new insight into the dissociating effect of strontium on bone resorption and formation. Acta Biomater. 2011;7(6):2593-2603.

25. Saidak Z, Marie PJ. Strontium signaling: molecular mechanisms and therapeutic implications in osteoporosis. Pharmacol Ther. 2012; 136(2):216-226.

26. Yang F, Yang D, Tu J, Zheng Q, Cai L, Wang L. Strontium enhances osteogenic differentiation of mesenchymal stem cells and in vivo bone formation by activating Wnt/catenin signaling. Stem Cells. 2011;29(6): 981-991.

27. Peng S, Liu XS, Huang S, et al. The cross-talk between osteoclasts and osteoblasts in response to strontium treatment: involvement of osteoprotegerin. Bone. 2011;49(6):1290-1298.

28. Li Y, Li Q, Zhu S, et al. The effect of strontium-substituted hydroxyapatite coating on implant fixation in ovariectomized rats. Biomaterials. 2010;31(34):9006-9014.

29. Maimoun L, Brennan TC, Badoud I, Dubois-Ferriere V, Rizzoli R, Ammann P. Strontium ranelate improves implant osseointegration. Bone. 2010;46(5):1436-1441.

30. Li Y, Li X, Song G, Chen K, Yin G, Hu J. Effects of strontium ranelate on osseointegration of titanium implant in osteoporotic rats. Clin Oral Implants Res. 2012;23(9):1038-1044.

31. Andersen OZ, Offermanns V, Sillassen M, et al. Accelerated bone ingrowth by local delivery of strontium from surface functionalized titanium implants. Biomaterials. 2013;34(24):5883-5890.
32. Newman SD, Lotfibakhshaiesh N, O’Donnell M, et al. Enhanced osseous implant fixation with strontium-substituted bioactive glass coating. Tissue Eng Part A. 2014;20(13-14):1850-1857.

33. Li Y, Qi Y, Gao Q, et al. Effects of a micro/nano rough strontium-loaded surface on osseointegration. Int J Nanomedicine. 2015;10:4549-4563.

34. Schumacher M, Lode A, Helth A, Gelinsky M. A novel strontium(II)modified calcium phosphate bone cement stimulates human-bonemarrow-derived mesenchymal stem cell proliferation and osteogenic differentiation in vitro. Acta Biomater. 2013;9(12):9547-9557.

35. Chou J, Valenzuela SM, Santos J, etal. Strontium-and magnesium-enriched biomimetic beta-TCP macrospheres with potential for bone tissue morphogenesis. J Tissue Eng Regen Med. 2014;8(10):771-778.

36. Santocildes-Romero ME, Crawford A, Hatton PV, Goodchild RL, Reaney IM, Miller CA. The osteogenic response of mesenchymal stromal cells to strontium-substituted bioactive glasses. J Tissue Eng Regen Med. 2015;9(5):619-631.

37. Park JW, Kwon TG, Suh JY. The relative effect of surface strontium chemistry and super-hydrophilicity on the early osseointegration of moderately rough titanium surface in the rabbit femur. Clin Oral Implants Res. 2012;24(6):706-709.

38. Offermanns V, Andersen OZ, Falkensammer G, et al. Enhanced osseointegration of endosseous implants by predictable sustained release properties of strontium. J Biomed Mater Res B Appl Biomater. 2015; 103(5):1099-1106.

39. Stadlinger B, Korn P, Tödtmann N, et al. Osseointegration of biochemically modified implants in an osteoporosis rodent model. Eur Cell Mater. 2013;25:326-40; discussion 339-340.

40. Donath K, Breuner G. A method for the study of undecalcified bones and teeth with attached soft tissues. The Sage-Schliff (sawing and grinding) technique. J Oral Pathol. 1982;11(4):318-326.

41. Sillassen M, Jeppesen CS, Andersen OZ, et al. Controlled Sr release from $\mathrm{Ti}-\mathrm{Sr}-\mathrm{O}$ films deposited by non-reactive magnetron sputtering in an industrial setup. Surf Coat Technol. 2014;252:56-63.

42. Jeffcoat MK, Chesnut CH 3rd. Systemic osteoporosis and oral bone loss: evidence shows increased risk factors. J Am Dent Assoc. 1993;124(11):49-56.

43. August M, Chung K, Chang Y, Glowacki J. Influence of estrogen status on endosseous implant osseointegration. J Oral Maxillofac Surg. 2001;59(11):1285-1289; discussion 1290-1291.

44. Piarulli G, Rossi A, Zatti G. Osseointegration in the elderly. Aging Clin Exp Res. 2013;25(1):S59-S60.

45. Wu C, Ramaswamy Y, Kwik D, Zreiqat H. The effect of strontium incorporation into $\mathrm{CaSiO} 3$ ceramics on their physical and biological properties. Biomaterials. 2007;28(21):3171-3181.

46. Saidak Z, Haÿ E, Marty C, Barbara A, Marie PJ. Strontium ranelate rebalances bone marrow adipogenesis and osteoblastogenesis in senescent osteopenic mice through NFATc/Maf and Wnt signaling. Aging Cell. 2012;11(3):467-474.

47. Meunier PJ, Slosman DO, Delmas PD, et al. Strontium ranelate: dose-dependent effects in established postmenopausal vertebral osteoporosis - a 2-year randomized placebo controlled trial. J Clin Endocrinol Metab. 2002;87(5):2060-2066.

48. Habibovic P, de Groot K. Osteoinductive biomaterials - properties and relevance in bone repair. J Tissue Eng Regen Med. 2007;1(1):25-32.

49. Haidar ZS, Hamdy RC, Tabrizian M. Delivery of recombinant bone morphogenetic proteins for bone regeneration and repair. Part A: current challenges in BMP delivery. Biotechnol Lett. 2009;31(12): 1817-1824.

50. Kloss FR, Singh S, Hächl O, et al. BMP-2 immobilized on nanocrystalline diamond-coated titanium screws; demonstration of osteoinductive properties in irradiated bone. Head Neck. 2013;35(2):235-241.

51. Noble PC, Box GG, Kamaric E, Fink MJ, Alexander JW, Tullos HS. The effect of aging on the shape of the proximal femur. Clin Orthop Relat Res. 1995;(316):31-44.

52. Moritz N, Alm JJ, Lankinen P, Mäkinen TJ, Mattila K, Aro HT. Quality of intertrochanteric cancellous bone as predictor of femoral stem RSA migration in cementless total hip arthroplasty. J Biomech. 2011; 44(2):221-227. 


\section{Publish your work in this journal}

The International Journal of Nanomedicine is an international, peerreviewed journal focusing on the application of nanotechnology in diagnostics, therapeutics, and drug delivery systems throughout the biomedical field. This journal is indexed on PubMed Central, MedLine, CAS, SciSearch $\AA$, Current Contents $\AA /$ Clinical Medicine,
Journal Citation Reports/Science Edition, EMBase, Scopus and the Elsevier Bibliographic databases. The manuscript management system is completely online and includes a very quick and fair peer-review system, which is all easy to use. Visit http://www.dovepress.com/ testimonials.php to read real quotes from published authors.

Submit your manuscript here: http://www.dovepress.com/international-journal-of-nanomedicine-journal 